\title{
ON A VARIETAL STRUCTURE OF ALGEBRAS
}

\author{
BY
}

R. D. GIRI

ABSTRACT. Shafaat introduced two successive generalisations of the variety of algebras: namely the semivariety and the quasivariety. We study a slightly more generalised concept which we call a pseudovariety.

1. Introduction. Quasiprimitive classes of algebras were characterized by Isbell [3] as the category of algebras closed under the formation of subalgebras, isomorphs and cartesian products. Shafaat [9] further divided them into two subclasses: (i) uniform quasiprimitive classes and (ii) nonuniform quasiprimitive classes.

A quasiprimitive class $\underline{\underline{C}}$ is called uniform, if there exists a cardinal $N$ such that an algebra $A \in \underline{\underline{C}}$ iff every subalgebra of $A$ generated by $N$ elements is in $\underline{\underline{C}}$. Otherwise we call $\underline{\underline{C}}$ nonuniform. Shafaat [7] called these uniform quasiprimitive classes implicationally defined (or simply implicational) classes of algebras, because they are characterizable by a set of implications. Since all the known examples of implicational classes, say variety, semivariety and quasivariety etc., end with the term 'variety', we assign the name varietal structure to an implicational class.

We study here a natural generalisation of the concept of a quasivariety which we call a pseudovariety. Let $w_{i}, w_{i}^{\prime}, w$ and $w^{\prime}$ be $\Omega$-words (i.e. elements of the word algebra $W_{\Omega}(X)$ of some arbitrary set $X$ (see [7, p. 137]). Then formulae $w_{i}=w_{i}^{\prime}$ and $w=w^{\prime}$ are called equations. A sentence of the form

$$
\forall X\left[\left(w_{1}=w_{1}^{\prime} \wedge \cdots \wedge w_{m}=w_{m}^{\prime}\right) \rightarrow w=w^{\prime}\right]
$$

is called an identical implication. The part on the left of the arrow $(\rightarrow)$ is called the 'data' and that on the right of ' $\rightarrow$ ' is called the 'consequence'. Omitting quantifiers and brackets we write

$$
w_{1}=w_{1}^{\prime} \wedge \cdots \wedge w_{m}=w_{m}^{\prime} \rightarrow w=w^{\prime} .
$$

Let $R_{1}$ and $R_{2}$ denote the sets of 'data' and 'consequence' respectively. Then a statement $\forall X\left(R_{1} \rightarrow R_{2}\right)$ may be interpreted: every solution of $R_{1}$ is also a

Received by the editors November $9,1973$.

AMS (MOS) subject classifications (1970). Primary 08A15; Secondary 08A25, 18A20, 18A30, 06A20.

Key words and phrases. Implicational class, implications, pseudovariety, varietal structure, countable character, inverse limit, full subcategory, finite basis property, lattice. 
solution of $R_{2}$. The length of an implication is defined as the cardinality of $R_{1}$.

REMARK 1.1. Identities (namely identical relations) are implications of length zero, that is, $R_{1}=\varnothing$. Equivalently identities consist only of the consequence part of the form $w=w^{\prime}$. However, they can be regarded as implications (of any finite length) with the data consisting of sufficiently many equations of the form $v=v$.

DEFINITION 1.2. The implicational length of a varietal structure is defined to be the least cardinal $N$ such that it may be defined by implications of length not exceeding $N$. Thus the implicational length of a variety is zero and of a semivariety is one, while a quasivariety has finite implicational length (see [6], [7], [11] and [12]).

DEFINITION 1.3. A varietal structure is called a pseudovariety if it may be defined by implications of at most countable length. We refer to $\S 3$ for its examples.

REMARK 1.4. Clearly

varieties $\subset$ semivarieties $\subset$ quasivarieties $\subset$ pseudovarieties.

2. Notation. Doubly underlined Latin letters denote classes of algebras, categories or quasiprimitive classes of algebras. $Q(\underline{\underline{K}})$ denotes the quasiprimitive class generated by $\underline{\underline{K}}$, that is, the class of all algebras that are embeddable in cartesian products of algebras of $\underline{\underline{K}}$.

Ob $\underline{\underline{C}}$ is the class of objects of category $\underline{\underline{C}}$.

$W_{\Omega}(X)$ is the $\Omega$-word algebra over an arbitrary set $X$ (cf. [1, p. 116]).

$L_{C}(X)$ is the lattice of congruences $\rho$ over $W_{\Omega}(X)$ such that $w_{\Omega}(X) / \rho$ is an algebra of $\underline{\underline{C}}$.

$\operatorname{alg}\left(l_{1}, l_{2}, \ldots\right)$ stands for the algebra generated by $l_{1}, l_{2}, \ldots$

laws $\underline{\underline{V}}$ denotes the set of all laws satisfied in variety $\underline{\underline{V}}$.

imp $\underline{\underline{K}}$ stands for the set of all implications satisfied in varietal structure $\underline{\underline{K}}$.

$T$-ideal is a two-sided ideal which is mapped into itself by every endomorphism of $W_{\Omega}(X)$.

$S_{\mathrm{V}}(L)$ is the lattice of all join subsemilattices(1) of the lattice $L$.

3. Examples.

3.1. Varieties, semivarieties and quasivarieties are examples of pseudovarieties. But here we give some examples of pseudovariety which are not quasivarieties.

3.2. The classes of all residually finite groups in a variety form an example of a pseudovariety (cf. [5, Theorem 4]).

(1) A join (meet) subsemilattice of a lattice $(L ; V, \Lambda)$ is a subset of $L$ closed under finite joins (meets). A join (meet) subsemilattice will be called complete if it is closed under infinite joins (meets). 
3.3. All quasiprimitive classes of abelian groups are pseudovarieties but not all of them are quasivarieties (cf. [9, Theorem 4]).

3.4. Let $\underline{V}_{0} \subseteq \underline{V}_{1} \subseteq \cdots$ be an ascending sequence of varieties of groups and $\underline{\underline{U}}=\bigvee \underline{\underline{V}} i$ be their union. Then $\underline{\underline{U}}$ is a pseudovariety (cf. [5, Theorem 2]).

3.5. Let $S$ be the countable set of finite groups. Then $Q(S)$ is a pseudovariety.

Proof. $Q(S)$ is clearly the class of all residually finite groups and so, by 3.2 , is a pseudovariety.

4. Some properties. In this section, we shall discuss some properties of a pseudovariety. As is well known, a class $\underline{\underline{C}}$ is said to have local character (cf. Neumann [5]) provided an algebra $A \in \underline{\underline{C}}$ iff every finitely generated subalgebra of $A$ is in $C$. If we replace the term finitely generated by countably generated we say that class $\underline{\underline{C}}$ is of countable character. Further we know that quasiprimitive subcategories of quasiprimitive categories are quasiprimitive categories (cf. Shafaat [9]). Using this fact in the definitions of local and countable character, we conclude that quasivarieties are of local character and pseudovarieties are of countable character.

LEMMA 4.1. If $\underline{\underline{K}}$ is a varietal structure of countable character then so is $Q(\underline{\underline{K}})$.

Proof. This is trivial since $\underline{\underline{K}}=Q(\underline{\underline{K}})$ by definition, provided $\underline{\underline{K}}$ itself is quasiprimitive.

THEOREM 4.2. A class $\underline{K}$ of algebras is a pseudovariety iff $\underline{K}$ is a varietal structure of countable character.

Proof. The direct part is obvious. For the converse part suppose $\underline{K}$ is not a pseudovariety. Then $\underline{=}$ satisfies implications of the form

(a) $\quad \forall x_{1}, \ldots, x_{n}\left[\left(w_{1}=w_{1}^{\prime} \wedge \cdots \wedge w_{m}=w_{m}^{\prime} \wedge \cdots\right) \rightarrow w=w^{\prime}\right]$

where the $w_{i}$ 's etc. are the elements of the word algebra $w_{\Omega}\left(x_{1}, x_{2}, \ldots, x_{n}\right)$ and the length of $(\alpha)$ is uncountably infinite.

Let $R$ be an uncountable algebra of real numbers such that $R \notin \underline{K}$. Consider at most a countable number of values $l_{1}, l_{2}, \ldots$ of the words $w_{1}, w_{2}, \ldots$ respectively in $R$. Then $\operatorname{alg}\left(l_{1}, l_{2}, \ldots\right)=S$ is a countably generated subalgebra of $R$. Clearly $S \in K$ because the countable union of countable sets is countable. Thus there are algebras not in $K$ and all of whose countably generated subalgebras are in $\underline{K}$. Hence $\underline{K}$ is not of countable character, which leads to a contradiction to our assumption.

THEOREM 4.3. Let $K$ be a varietal structure of algebras all of whose subvarietal structures containing $\underline{\underline{K}}$ are pseudovarieties then $\underline{\underline{K}}$ is a pseudovariety. 
Proof. Clearly $\underline{\underline{K}}$ is the smallest subclass containing $\underline{\underline{K}}$, which is, a fortiori, the pseudovariety.

REMARK. This theorem cannot be generalized as: if all subvarietal structures of $\underline{\underline{K}}$ are pseudovarieties then so is $\underline{\underline{K}}$. It is, for $\underline{\underline{K}}$ may be chosen as a varietal structure defined by implicaitons of uncountably infinite length with all its sub-

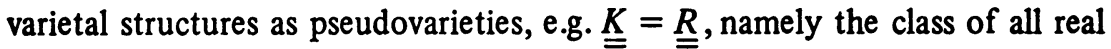
algebras.

THEOREM 4.4. If a pseudovariety $\underline{\underline{K}}$ has a countably infinite number of subpseudovarieties then every quasiprimitive subclass of $\underline{\underline{K}}$ is a pseudovariety.

ProOF. Suppose $\underline{\underline{Q}}$ is a quasiprimitive subclass of $\underline{\underline{K}}$ which cannot be defined by implications of countable length. Then $\underline{\underline{Q}}$ satisfies implications of the type

$$
\forall x_{1}, \ldots, x_{n}\left[\left(w_{1}=w_{1}^{\prime} \wedge \cdots \wedge w_{m}=w_{m}^{\prime} \wedge \cdots\right) \rightarrow w=w^{\prime}\right]
$$

where $(\alpha)$ has cardinality greater than or equal to $\aleph_{0}$, the $w_{i}^{\prime}$ s and $w_{i}^{\prime}$ 's are elements of the word algebra $W_{\Omega}\left(x_{1}, \ldots, x_{n}\right)$.

To each implication $(\alpha)$ of $\underline{\underline{Q}}$ we can associate uncountably many subpseudovarieties, because we can choose uncountably many sets $\Sigma_{i}$ of implications of countable length from $(\alpha)$. In this way $\underline{\underline{Q}}$ has uncountably many subpseudovarieties and so has $K$ itself. This contradicts the hypothesis. Hence the theorem is proved.

Through our study we came across a query:

QUERY 4.5. Let $K$ be a variety of $\Omega$-algebras such that every subpseudovariety of it is a quasivariety. Is every subvarietal structure of $\underline{\underline{K}}$ a quasivariety?

To this end, we note that a nonabelian, nilpotent variety $K$ of finite exponent $n(n>2)$ of groups is locally finite and has some nonresidually finite groups (cf. remark to Corollary 1 of Shafaat [10]). This further asserts the existence of infinitely many subquasivarieties of $\underline{=}$ by Corollary 1 of Shafaat [10].

Now the following example shows that the answer to our query is negative:

EXAMPLES 4.6. $\underline{\underline{K}}=\underline{\underline{N}}_{c} \wedge \underline{\underline{B}}_{4}$ is a variety of nilpotent goups of exponent 4 . This has infinitely many subquasivarieties $\underline{W}_{1}, \underline{W}_{2}, \ldots$ in view of the foregoing discussion. Clearly $\vee \underline{W}_{i}$ is a subvarietal structure of $\underline{K}$ which is not a quasivariety (see Example 3.4). However, every subpseudovariety (in fact subvariety) $\underline{N}_{c} \wedge$ $\underline{B}_{4 m}$ ( $m$ is a positive integer) of $\underset{\underline{K}}{=}$ is a quasivariety.

As in Shafaat [12], $\underline{\underline{V}}_{m, n}$ stands for the variety of algebras satisfying the system of identities

$$
\begin{array}{ll}
\phi_{i}\left(w_{1}\left(x_{1}, \ldots, x_{n}\right), \ldots, w_{m}\left(x_{1}, \ldots, x_{n}\right)\right)=x_{i}, & 1 \leqslant i \leqslant n, \\
w_{j}\left(\phi_{1}\left(x_{1}, \ldots, x_{m}\right), \ldots, \phi_{n}\left(x_{1}, \ldots, x_{m}\right)\right)=x_{j}, & 1 \leqslant j \leqslant m,
\end{array}
$$


where the $\phi_{i}$ 's are $n, m$-ary operations and $w_{j}$ 's are $m, n$-ary operations.

In Remark 3 of his paper [12] Shafaat proves that every subquasivariety of $\underline{\underline{V}}_{1, n}, n>1$, is a semivariety. But for pseudovarieties we are confronted with a problem:

PROBLEM 4.7. Is every subpseudovariety of variety $\underline{\underline{V}}_{m, n}$ of algebras, $m>$ $1, n \geqslant 1$, a quasivariety?

The answer to this problem seems to be negative but I do not find an example.

REMARK 4.8. In the case when $m=1, n \geqslant 1$ the answer is affirmative as $\underline{V}_{1, n}$ will have only a two element chain implying the existence of no proper subvariety (see, Introduction of [12]).

5. Categorical view. For basic terminology of universal algebra and category theory we refer the reader to Cohn [1] and Mitchell [4] respectively.

Our object is to prove two categorical characterisations of pseudovariety. We frequently use the ideas of Shafaat [9] and [7] in our arguments.

THEOREM 5.1. A full subcategory $K$ of $\underline{\underline{C}}$ is a pseudovariety iff

(1) for every set $X$ and $\rho \in L_{\underline{C}}(X)$, $\rho$ has a $L_{\underline{\underline{K}}}(X)$-cover,

(2) $A \in \mathrm{Ob} \underline{\underline{K}}$ iff every countably generated subalgebra of $A$ is in $\mathrm{Ob} \underline{\underline{K}}$.

ProOF. If $K$ is a pseudovariety then all the conditions are trivially satisfied. Conversely condition (1) ensures that $K$ is an implicational class (see [7, Theorem 1]). Condition (2) guarantees that $\underline{\bar{K}}$ is a pseudovariety by virtue of Theorem 4.2.

THEOREM 5.2. A full subcategory $\underline{\underline{K}}$ of $\underline{\underline{C}}$ is a pseudovariety iff

(a) $K$ is coreflective,

(b) for every monomorphism $A_{1} \rightarrow A_{2}$ in $\underline{\underline{C}}, A_{2} \in \underline{\underline{K}}$ implies $A_{1} \in \underline{\underline{K}}$. And inverse limit of countably finite or infinite directed downward system $\underline{\underline{D}} \subseteq \underline{\underline{K}}$ is again in $K$.

Proof. Condition (a) implies the condition (1) of Theorem 5.1 by virtue of Therorm 1 of Shafaat [7]. By taking countably finite or infinite directed downward systems with all maps monomorphic and then epimorphic in particular, we see that condition (b) satisfies (2) of Theorem 5.1. Hence $\underline{K}$ is a pseudovariety.

Conversely if $K$ is a pseudovariety defined by $\Sigma$ then $K$ is an implicational class which further implies that $K$ is coreflective by Theorem 1 of Shafaat [7]. Clearly since $K$ is a subalgebra closed, the first part of condition (b) is satisfied. For the second part, consider $Q=\Pi_{A \in D} A$ as the direct product in $\underline{\underline{C}}$ of all objects appearing in $\underline{\underline{D}}$. Let $\alpha_{A}: Q \rightarrow A$ be the projections and $\rho$ be the congru- 
ence over $A$ generated by $\left\{\left(q \alpha_{A}, q \alpha_{B} \cdot f\right) \mid f: B \rightarrow A, q \in Q\right\}$. Then $\beta_{A}$ : $\rho \stackrel{\iota}{\rightarrow} Q \stackrel{\alpha_{A}}{\rightarrow} A$ provides the inverse limit of $\underline{\underline{D}}$ where $\iota$ is the injection map. We need to show that $\rho \in \underline{\underline{K}}$.

Let $\left(w_{1}=w_{1}^{\prime} \wedge \cdots \wedge w_{m}=w_{m}^{\prime} \wedge \cdots\right) \rightarrow w=w^{\prime}$ be an implication in $\Sigma$ and $w_{i}\left(a_{1}, \ldots, a_{m}\right)=w_{i}^{\prime}\left(a_{1}, \ldots, a_{m}\right)$ be the word equations in $A, i$ varying over the set of natural numbers. Since $\alpha_{A}$ is projection and $D$ is directed downward we see $w_{i}\left(e_{1}, \ldots, e_{m}\right)=w_{i}^{\prime}\left(e_{1}, \ldots, e_{m}\right)$ is satisfied in $\rho$ where $a_{j} \beta_{A}^{-1}=$ $e_{j}, e_{j} \in \rho, 1 \leqslant j \leqslant m$. Since $A$ satisfies $\Sigma, w\left(e_{1}, \ldots, e_{m}\right)=w^{\prime}\left(e_{1}, \ldots, e_{m}\right)$ is valid in $\rho$. Hence the inverse limit $\rho$ of $\underline{\underline{D}}$ again lies in $\underline{\underline{K}}$.

6. Finite basis property. Shafaat [11] defines an implicational class $\underline{\underline{K}}$ to have finite basis property (fbp) for equations if within $\underline{\underline{K}}$ every system of equations in a finite number of variables is equivalent to a finite system in those variables. Alternatively, every congruence over a finitely generated (f.g.) algebra $A \in \underline{\underline{K}}$ is a f.g. subalgebra of $A \times A$. In still other terms every f.g. subalgebra of an algebra of $\underline{\underline{K}}$ is finitely presented.

Since every equation is an implication but not conversely, we can generalise the foregoing concept as follows:

A varietal structure $\underline{\underline{K}}$ has a finite basis property for its implications, if within $\underline{\underline{K}}$ every implication over a word algebra $W_{\Omega}(X)$ is derivable by a finite number of implications over $W_{\Omega}(X)$. Equivalently imp $K$ is a f.g. $T$-ideal of $W_{\Omega}(X)$. In still other terms every f.g. $T$-ideal of $W_{\Omega}(X)$ is a homomorphic image of imp $\underline{\underline{K}}$.

EXAMPLES. We give some examples of finitely based varietal structures from [11] and [6].

6.1. Every locally finite implicational class has fbp.

6.2. The class of all abelian groups has fbp for implications.

6.3. The class of commutative monoids has fbp for implications.

6.4. All finitely based varieties are examples of varietal structure with $\mathrm{fbp}$, e.g. nilpotent variety, metabelian variety, and Cross-variety, etc.

From [6] it is well-known that every subvariety of a variety $\underline{\underline{V}}$ is finitely based iff the maximum condition holds for fully invariant subgroups of the word group $X_{\infty}$ containing laws $\underline{\underline{V}}$. This observation can be generalised as follows:

Every subvarietal structure of a varietal structure $K$ is finitely based iff the maximum condition holds for $T$-ideals of $W_{\Omega}(X)$ containing imp $\underline{\underline{K}}$. Its proof can be formulated in a natural manner.

7. Lattices. For conceptual background we refer to Cohn [1] and Shafaat [12].

THEOREM 7.1. All pseudovarieties of solvable groups are quasivarieties and 
their lattice is isomorphic to the lattice $\left(S_{\mathrm{V}}, \leqslant,\left(Z^{+} \times 2\right)\right)$ of ideals of $Z^{+} \times 2$ where $Z^{+}$denotes the set of positive integers including a new zero, say 0 , under the order relation $\leqslant$ of division.

Proof. Similar to the symbol 0 in the lattice under consideration we can define a unit $\infty$.

Let $\underline{\underline{G}}$ be the class of groups and $M$ be the set of orders of finite cyclic groups in $\underline{\underline{G}}$. Assume $P=\{0, \infty\}$ or $\{0\}$ according as $\underline{\underline{G}}$ contains infinite cyclic groups or not. We define $|\underline{G}|$ as the ordered pair $\langle M, P\rangle$ and call it the order of

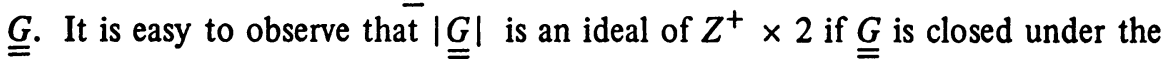
formation of subgroups and cartesian products.

Now we recall three well-known facts. Firstly, every abelian group is the union of a countable ascending chain of direct products of cyclic groups. Secondly, the union of a countable ascending chain of pseudovarieties is a pseudovariety. Thirdly, every solvable group of length $l$ is the $(l-1)$-step extension of abelian groups.

(*) Thus every pseudovariety of solvable groups consists of finite extensions of all the unions of countable ascending chains of direct products of its cyclic groups. Therefore, it can be determined by its order.

Suppose $M \cup P=N$. Let $\Sigma(\underline{\underline{G}})$ be the set of implications $\sigma_{n}: x^{n}=1 \rightarrow$ $x^{\theta(n)}=1, n \in N, \theta(n)$ is the largest number in $N$ but not exceeding $n$. It is easy to prove (cf. Proof of Theorem 1 in Shafaat [12]) that the order

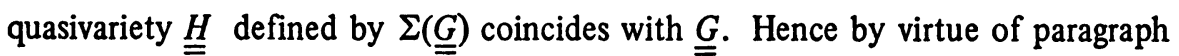
(*) $\underline{\underline{G}}=\underline{\underline{H}}$. This proves the theorem.

Acknowledgement. I would like to express my heartfelt gratitude to Dr. Sheila Oates Macdonald (Brisbane) for her valuable suggestions. I also wish to acknowledge the comments of the referee and the encouragement of my supervisor Professor M. A. Kazim.

\section{REFERENCES}

1. P. M. Cohn, Universal algebra, Harper \& Row, New York and London, 1965. MR 31 \#224.

2. J. A. Gerhard and Ahmad Shafaat, Semivarieties of idempotent semigroups, Proc. London Math. Soc. (3) 22 (1971), 667-680. MR 45 \#2048.

3. J. R. Isbell, Subobjects, adequacy, completeness and categories of algebras, Rozprawy Mat. 36 (1964). MR 29 \#1238.

4. B. Mitchell, Theory of categories, Pure and Appl Math., vol. 17, Academic Press, New York and London, 1965. MR 34 \#2647.

5. B. H. Neumann, Groups properties with countable character (unpublished).

6. Hanna Neumann, Varieties of groups, Ergebnisse der Mathematik und ihrer Grenzgebiete, Band 37, Springer-Verlag, New York, 1967. MR 35 \#6734.

7. Ahmad Shafaat, On implicationally defined classes of algebras, J. London Math. Soc. 44 (1969), 137-140. MR 38 \#5691.

8. - On the structure of certain idempotent semigroups, Trans. Amer. Math. Soc. 149 (1970), 371-378. MR 41 \#3640. 
9. Ahmad Shafaat, $A$ note on quasiprimitive classes of algebras, J. London Math. Soc. (2) 2 (1970), 489-492. MR 43 \#1914.

10. - Subcartesian products of finitely many finite algebras, Proc. Amer. Math. Soc. 26 (1970), $401-404$. MR 42 \#172.

11. - Remarks on quasivarieties of algebras (unpublished).

12. - Lattices of subsemivarieties of certain varieties, J. Austral. Math. Soc. 12 (1971), 15-20. MR 43 \#1906.

DEPARTMENT OF MATHEMATICS, ALIGARH MUSLIM UNIVERSITY, ALIGARH, INDIA 